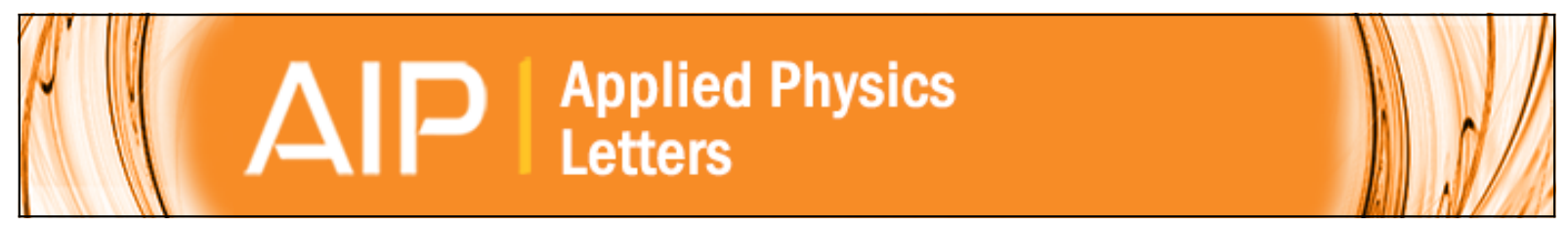

\title{
Simple liquid-core waveguide polarimetry
}

Thomas C. Preston, Nathan D. Jones, Sven Stille, and Silvia Mittler

Citation: Applied Physics Letters 89, 253509 (2006); doi: 10.1063/1.2404591

View online: http://dx.doi.org/10.1063/1.2404591

View Table of Contents: http://scitation.aip.org/content/aip/journal/apl/89/25?ver=pdfcov

Published by the AIP Publishing

\section{Articles you may be interested in}

Chiral cavity ring down polarimetry: Chirality and magnetometry measurements using signal reversals

J. Chem. Phys. 143, 104202 (2015); 10.1063/1.4930109

Shot-noise-limited optical Faraday polarimetry with enhanced laser noise cancelling

J. Appl. Phys. 115, 103101 (2014); 10.1063/1.4867743

Liquid-core low-refractive-index-contrast Bragg fiber sensor

Appl. Phys. Lett. 98, 201114 (2011); 10.1063/1.3592758

Polarimetry in turbid, birefringent, optically active media: A Monte Carlo study of Mueller matrix decomposition in the backscattering geometry

J. Appl. Phys. 105, 102023 (2009); 10.1063/1.3116129

A frequency stabilization method for diode lasers utilizing low-field Faraday polarimetry

Rev. Sci. Instrum. 76, 093108 (2005); 10.1063/1.2038305

\section{AlP $\mid$ APL Photonics}

APL Photonics is pleased to announce Benjamin Eggleton as its Editor-in-Chief

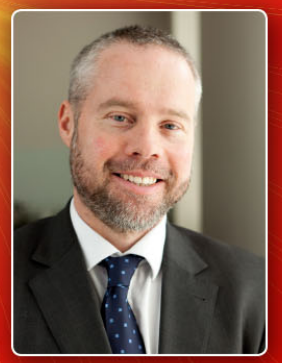




\title{
Simple liquid-core waveguide polarimetry
}

\author{
Thomas C. Preston and Nathan D. Jones \\ Department of Chemistry, The University of Western Ontario, London, Ontario N6A 5B7, Canada
}

\author{
Sven Stille and Silvia Mittler ${ }^{a}$ \\ Department of Physics and Astronomy, The University of Western Ontario, London, \\ Ontario N6A 3K7, Canada
}

(Received 30 August 2006; accepted 6 November 2006; published online 20 December 2006)

\begin{abstract}
A simple, low cost, liquid-core waveguide polarimeter is presented. The rotation of polarized light was measured after passing through a hollow fiber containing the chiral solution of interest. Sensitivity was found to scale with fiber length. For compactness, the fiber can be curled into a right-angle figure 8 configuration and this eliminated any phase effects that would otherwise be introduced. The limit of detection was $0.3 \mathrm{mM}$ for both $(S)-(-)$-limonene and $(R)-(+)$-limonene using a $220.8 \mathrm{~cm}$ long fiber with a run time of approximately $30 \mathrm{~s}$ and a core volume of $38.9 \mu \mathrm{l}$.

(C) 2006 American Institute of Physics. [DOI: 10.1063/1.2404591]
\end{abstract}

The rotation of polarized light is considered to be the defining physical property of chiral molecules. These nonsuperimposable mirror images will rotate polarized light in opposite directions and this has historically been important in recognizing the excess of one enantiomer over the other in scalemic mixtures. As a quantitative method, however, polarimetry suffers from serious drawbacks. Optical rotation is typically a very weak effect and this leads to poor sensitivity and the need to increase interrogation path lengths and, consequently, sample volumes in order to compensate. Furthermore, the general lack of selectivity limits its applicability when chiral contaminants are present in solution. ${ }^{1}$

This has lead to the development of a series of alternative approaches to enantiomeric screening which span most areas of analytical chemistry. ${ }^{2}$ However, polarimetry has far from been abandoned and recent demonstrations of gas phase cavity ring-down polarimetry, ${ }^{3,4}$ frequency domain polarimetry, ${ }^{5}$ and measurements of nanoliter volumes using capillary interferometry ${ }^{6}$ illustrate the continued appeal of the technique largely due to its conceptual simplicity and its applicability to all chiral molecules.

In this letter we present an approach yet to be taken: a polarimeter built using liquid-core waveguides. The optics of these waveguides are not different from those of conventional optical fibers: light propagates through a high-index core that is surrounded by a cladding of lower index. This is the well-known phenomenon of total internal reflection. ${ }^{7}$ Here, the cladding is a low transmission loss hollow fiber and the core is a solvent of higher index containing the analyte of interest. In a conventional polarimeter, linear polarized light rotates as it passes through a cuvette containing the sample under study. ${ }^{8}$ In a liquid-core waveguide, rotation would occur as light propagates through the core; therefore, in a sense, the hollow fiber is analogous to the cuvette and, likewise, the core is no different from the sample that fills it. With this system, measurements could be taken by coupling light in at one end of the waveguide and determining the rotation at the opposite end. The advantage of this would be that, similar to other liquid-core waveguides, the scalability of path length allows for large sensitivity enhancements with

${ }^{a}$ Author to whom correspondence should be addressed; electronic mail: smittler@uwo.ca little or no increase to instrument size or cost. ${ }^{9}$ Furthermore, by their very design, these waveguides are readily incorporated into rapid screening systems. Injection and pumping are required during the filling process and this can easily be automated to handle a large series of samples.

A hollow core fused silica fiber with a $150 \mu \mathrm{m}$ inner diameter and a $363 \mu \mathrm{m}$ outer diameter (TSP150375, Polymicro Technologies, Phoenix, Arizona) was mounted into the setup shown in Fig. 1. Light from a cw linearly polarized He-Ne laser of either $\lambda=543.5 \mathrm{~nm}$ (Research Electro-Optics, $0.5 \mathrm{~mW}$ ) or $\lambda=632.8 \mathrm{~nm}$ (Research Electro-Optics, $12.0 \mathrm{~mW}$ ) was coupled into the waveguide with a microscope objective $(10 \times$, numerical aperture $=0.25, f$ $=16.5 \mathrm{~mm}$, Newport).

The emerging polarization was measured using one of two setups: the beam splitter arrangement shown in Fig. 1 or, alternatively, with a computer-controlled rotating polarizer that scanned through $180^{\circ}$ generating a Malus curve (this required only one detector and the beam splitter was removed from the optical train). Typically, this second setup was used (i) to ensure that the light propagating through the fiber was linear before other experiments were run and (ii) to investigate other polarization effects besides optical rotation that may occur in the fiber. However, once the fiber had been well characterized, the beam splitter arrangement was more advantageous mainly because it allowed for simple corrections to intensity fluctuations that may have occurred as light propagated through the fiber and, therefore, sped up data analysis.

To understand how this is accomplished, first consider the propagation of light in Fig. 1 using the following Jones calculus representation: ${ }^{10}$

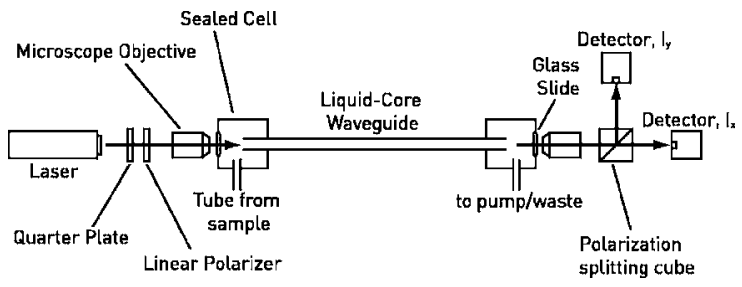

FIG. 1. Experimental setup of polarimeter. 
TABLE I. Specific rotations and limits of detection (LOD) (calculated using $2 \sigma /$ slope, $\sigma$ was determined over 20 measurements) for chiral species at the wavelengths of 543 and $632.8 \mathrm{~nm}$ and fiber lengths of 26.3 and $220.8 \mathrm{~cm}$ (Curled in a RAF8 configuration with two loops of equal radii). The $1 \mathrm{~cm}$ cell constants were determined with a conventional polarimetry experiment and are listed here for reference. All measurements were made at $25^{\circ} \mathrm{C}$.

\begin{tabular}{|c|c|c|c|c|c|}
\hline & \multicolumn{3}{|c|}{ Specific rotation $[\alpha]\left(10^{-1} \mathrm{~cm}^{2} \mathrm{~g}^{-1}\right)$} & \multicolumn{2}{|c|}{ LOD (mM) } \\
\hline & $\begin{array}{l}1 \mathrm{~cm} \\
\text { cell }\end{array}$ & $\begin{array}{c}26.3 \mathrm{~cm} \\
\text { fiber }\end{array}$ & $\begin{array}{l}220.8 \mathrm{~cm} \\
\text { fiber }\end{array}$ & $\begin{array}{c}26.3 \mathrm{~cm} \\
\text { fiber }\end{array}$ & $\begin{array}{c}220.8 \mathrm{~cm} \\
\text { fiber }\end{array}$ \\
\hline $\begin{array}{l}\lambda=543.5 \mathrm{~nm} \\
(R)-(+) \text {-limonene } \\
(S)-(-) \text {-limonene } \\
(R)-(-) \text {-carvone } \\
(S)-(+) \text {-carvone } \\
(1 R, 5 R)-(+)-\alpha \text {-pinene } \\
(1 S, 5 S)-(-)-\alpha \text {-pinene }\end{array}$ & $\begin{array}{c}147 \pm 1 \\
-129 \pm 1 \\
-75 \pm 1 \\
76 \pm 1 \\
59 \pm 1 \\
-58 \pm 1\end{array}$ & $\begin{array}{c}148 \pm 3 \\
-122 \pm 5 \\
-65 \pm 3 \\
74 \pm 5 \\
66 \pm 3 \\
-57 \pm 2\end{array}$ & $\begin{array}{c}145.0 \pm 2.4 \\
-130.4 \pm 1.3 \\
-65.9 \pm 1.5 \\
66.5 \pm 1.4 \\
61.9 \pm 0.7 \\
-59.9 \pm 0.7\end{array}$ & $\begin{array}{l}3 \\
3 \\
5 \\
5 \\
6 \\
7\end{array}$ & $\begin{array}{l}0.3 \\
0.3 \\
0.6 \\
0.6 \\
0.7 \\
0.8\end{array}$ \\
\hline $\begin{array}{l}\lambda=632.8 \mathrm{~nm} \\
(R)-(+)-\text { limonene } \\
(S)-(-) \text {-limonene } \\
(R)-(-) \text {-carvone } \\
(S-(+)) \text {-carvone } \\
(1 R, 5 R)-(+)-\alpha \text {-pinene } \\
(1 S, 5 S)-(-)-\alpha \text {-pinene }\end{array}$ & $\begin{array}{c}104 \pm 1 \\
-92 \pm 1 \\
-52 \pm 1 \\
53 \pm 1 \\
44 \pm 1 \\
-41 \pm 1\end{array}$ & $\begin{array}{c}105 \pm 2 \\
-100 \pm 2 \\
-51 \pm 2 \\
50 \pm 3 \\
41 \pm 2 \\
-46 \pm 4\end{array}$ & $\begin{array}{c}103.8 \pm 1.0 \\
-93.2 \pm 0.5 \\
-44.8 \pm 0.5 \\
46.1 \pm 0.3 \\
43.1 \pm 0.3 \\
-42.8 \pm 0.1\end{array}$ & $\begin{array}{c}5 \\
5 \\
9 \\
9 \\
13 \\
11\end{array}$ & $\begin{array}{l}0.7 \\
0.8 \\
1.4 \\
1.4 \\
1.6 \\
1.6\end{array}$ \\
\hline
\end{tabular}

$$
\left(\begin{array}{l}
E_{x} \\
E_{y}
\end{array}\right)=\left(\begin{array}{cc}
\cos \theta & -\sin \theta \\
\sin \theta & \cos \theta
\end{array}\right)\left(\begin{array}{c}
\cos \phi \\
\sin \phi
\end{array}\right) E_{0},
$$

where $\theta$ is the angle of rotation that results from optical activity present in the core, $\phi$ is the angle of the linear polarizer that follows the quarter plate, and $E_{0}$ is the time dependent amplitude of the field emerging from that polarizer. The limitation of Eq. (1) is that it cannot be applied to fibers where phase effects, such as retardation caused by a stressinduced birefringence, are present. A second possible limitation would be the presence of linear turning effects that are artifacts of the fiber itself. This can easily be overcome, however, by comparing all measurements to a reference (e.g., a measurement made with a nonoptically active core).

Since the two detectors measure intensity, Eq. (1) must be multiplied by its complex conjugate to be useful. This yields an equation that relates rotation to what is physically measured,

$\left(\begin{array}{l}I_{x} \\ I_{y}\end{array}\right)=\left(\begin{array}{c}\cos ^{2} \theta \cos ^{2} \phi-2 \cos \theta \cos \phi \sin \theta \cos \phi+\sin ^{2} \theta \cos ^{2} \phi \\ \sin ^{2} \theta \cos ^{2} \phi+2 \sin \theta \cos \phi \cos \theta \sin \phi+\cos ^{2} \theta \sin ^{2} \phi\end{array}\right) I_{0}$.

The convenience of Eq. (2) is that when it is represented in the following form:

$$
\frac{I_{x}-I_{y}}{I_{x}+I_{y}}
$$

simplification allows for rotation to be solved easily as a function of the two measured intensities and the entrance angle $\phi$;

$$
\cos (2 \theta+2 \phi)=\frac{I_{x}-I_{y}}{I_{x}+I_{y}} .
$$

Furthermore, the denominator on the right side of Eq. (4) normalizes for any intensity fluctuations that may occur as the light propagates through the fiber. ${ }^{7}$

In order to explore how optical activity affects the rotation of polarized light in our liquid-core waveguide, several chiral compounds with different optical activities were studied. $(R)-(+)$-limonene, $(S)-(-)$-limonene, $(R)-(-)$-carvone,
$(S)-(+)$-carvone, $(1 R, 5 R)-(+)-\alpha$-pinene, and $(1 S, 5 S)-(-)-\alpha$ -pinene were all purchased from Sigma-Aldrich and prepared as serial dilutions in toluene.

Optical rotation was measured at the wavelengths of 543.5 and $632.8 \mathrm{~nm}$ and the specific rotation, $[\alpha]_{\lambda}^{25^{\circ} \mathrm{C}}$, was calculated using

$$
[\alpha]_{\lambda}^{25^{\circ} \mathrm{C}}=\frac{\theta}{l c},
$$

where $\theta$ is the observed rotation in degrees [from Eq. (4)], $l$ is the path length in $\mathrm{dm}$, and $c$ is the concentration in $\mathrm{g} / \mathrm{mL}$. However, a more convenient form of Eq. (5) is

$$
[\alpha]_{\lambda}^{25^{\circ} \mathrm{C}}=\frac{10^{7} \theta}{l^{\prime} c^{\prime} \mathrm{MW}},
$$

where $l^{\prime}$ is the path length in $\mathrm{cm}, c^{\prime}$ is the concentration in $\mathrm{mM}$, and $\mathrm{MW}$ is the molecular weight in $\mathrm{g} / \mathrm{mol}$.

For reference, a conventional polarimetry experiment was performed using the neat chemicals in $1 \mathrm{~cm}$ path length UV/vis cells. This experiment simply involved measuring the rotation of linear polarized light after passing through the cell with the polarization sensitive cube setup described above. These values are listed in Table I.

Differences in the optical constants calculated at the two different wavelengths arise due to optical rotatory dispersion. Additionally, $(R)-(+)$-limonene consistently showed an optical constant greater than that of $(S)-(-)$-limonene. This is related to the purity of the chemicals themselves, not the polarimetry technique.

The results for running the chiral solutions through a linear, $26.3 \mathrm{~cm}$ long fiber are shown in Fig. 2 and Table I for both laser wavelengths. These not only indicate the expected linear relationship between rotation and concentration but also show that the path length term in Eq. (5) can simply be replaced by fiber length when calculating optical constants.

This second result, the equivalence of path length to fiber length, highlights the potential for using a liquid-core waveguide in polarimetry: as the length of the capillary is 


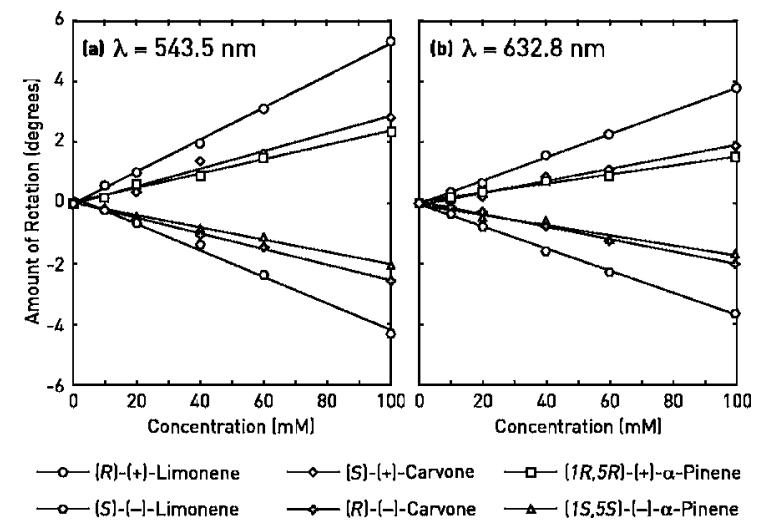

FIG. 2. Rotation angle vs concentration of three enantiomeric pairs $[(R)$ - $(+)$-limonene, $(S)$-(-)-limonene, $(R)-(-)$-carvone, $(S)-(+)$-carvone, $(1 R, 5 R)-(+)$ - $\alpha$-pinene, and $(1 S, 5 S)-(-)-\alpha$-pinene $]$ in a linear fiber of $26.3 \mathrm{~cm}$ in length [(a) for $\lambda=543.5 \mathrm{~nm}$ and (b) for $\lambda=632.8 \mathrm{~nm}$ ].

increased, sensitivity should increase proportionally. Furthermore, because light propagates through total internal reflection, there should be little variation in the intensity of the transmitted beam. In addition, the small diameter of the capillary means that the volume of sample required to fill the fiber will always be very small compared to conventional cuvettes. For a fiber length of $26.3 \mathrm{~cm}$, the volume of the core is $4.65 \mu \mathrm{l}$; for $220.8 \mathrm{~cm}$, it is $38.9 \mu \mathrm{l}$. Using a peristaltic pump (EV 500, Autoclude, $60 \mathrm{ml} / \mathrm{min}$ ), the fill times for these two fibers were less than a second and around $30 \mathrm{~s}$, respectively. Finally, the small changes to the refractive index of the core that occurred while running samples of different compositions and concentrations did not appear to influence the observed rotation.

Extending the fiber to longer lengths will eventually require that it be curled. It is well known that such an action introduces a birefringence in the form of perpendicular "fast" and "slow" planes. ${ }^{7}$ This effect is most easily seen when the angle of polarized light entering the fiber is varied while the optical activity of the core remains constant (Fig. 3, double loop plot). These periodic deviations will obscure the true rotation of polarized light by any optically active species present in the fiber core and a fiber curled in this manner would be difficult to use in any practical application without extensive data analysis and calibration. However, by curling the fiber into two loops at right angles to each other and creating a right-angle figure 8 (RAF8) configuration, these deviations will be eliminated as the fast and slow planes will effectively cancel each other out over the total distance of the two loops (Fig. 3, RAF8 plot).

For chiral solutions run through a $220.8 \mathrm{~cm}$ fiber curled in a RAF8 configuration, the sensitivity was, again, found to be proportional to the fiber length; this resulted in an approximately eightfold gain over the $26.3 \mathrm{~cm}$ fiber (Table I).

With our simple experimental setup, observing changes as small as $90 \mathrm{mdeg}(\lambda=632.8 \mathrm{~nm})$ and $70 \mathrm{mdeg}(\lambda$ $=543.5 \mathrm{~nm}$ ) were found to be possible (the deviation, in millidegrees, over 20 measurements). This resolution, however, should not be used as a figure of merit when attempting a comparison to other polarimeters. Only a limit of detection (LOD) that takes into account the additional factors of the optical constant of the sample, the wavelength of light, and the path length of the polarimeter should be used for this

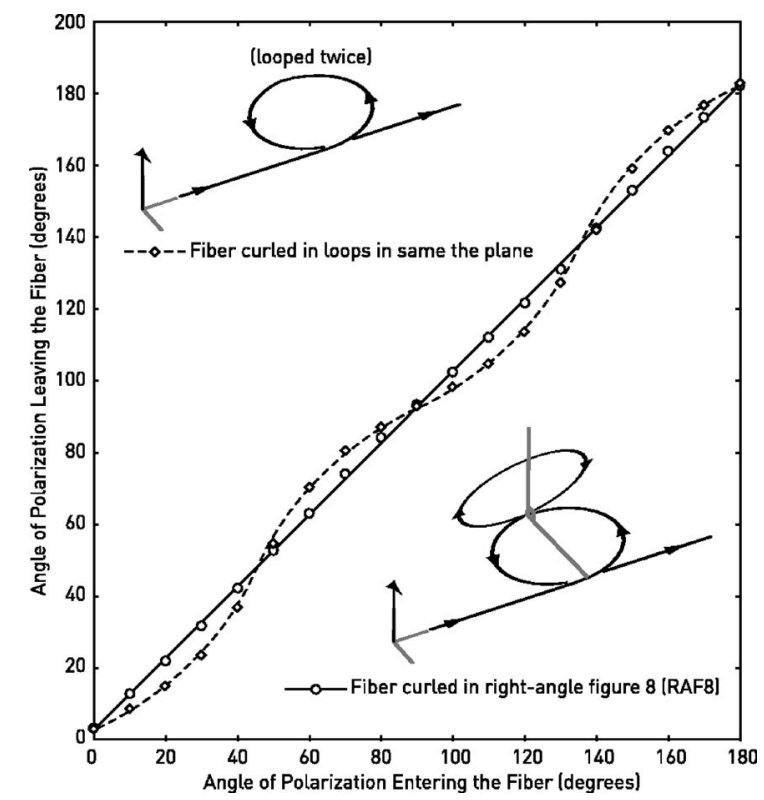

FIG. 3. Angle of polarization after passing through a fiber vs the angle of polarization entering a fiber for $\lambda=632.8 \mathrm{~nm}$ using a fiber length of $239.7 \mathrm{~cm}$ filled with toluene. The fiber was curled into either a double loop or a RAF8 configuration (see insets). The angle of $0^{\circ}$ is defined as being perpendicular to the plane of the first loop. The radii of all loops are equal $(17 \mathrm{~cm})$.

type of evaluation. Clearly, path length is the inherent advantage of our polarimeter, but as the technology matures improvements to the area of resolution should be possible. Using techniques not employed here (e.g., lock-in amplifiers), resolutions comparable to those of commercially available instruments (typically on the order of microdegrees) should be attainable and, consequently, a LOD of a few orders of magnitude greater than what has been reported here can be expected.

Mohammad Nuruzzaman and Uwe Langbein are thanked for useful discussions. Financial aid came from Emerging Materials Knowledge [EMK No. CA01085, Materials and Manufacturing Ontario and the Ontario Research, and Development Challenge Fund (ORDCF)], NSERC, CFI, OIF, and Ontario Photonics Consortium (through ORDCF). One of the authors (S.S.) thanks the University of Applied Sciences, Wiesbaden, Germany, for travel support and another author (S.M.) thanks the CRC Program of the Government of Canada.

${ }^{1}$ E. Charney, The Molecular Basis of Optical Activity (Wiley, New York, 1979), p. 41.

${ }^{2}$ M. G. Finn, Chirality 14, 534 (2002), and references therein.

${ }^{3}$ T. Muller, K. B. Wiberg, and P. H. Vaccaro, J. Phys. Chem. A 104, 5959 (2000).

${ }^{4}$ R. Engeln, G. Berden, E. van den Berg, and G. J. Meijer, Chem. Phys. 107, 4458 (1997).

${ }^{5}$ F. Vollmer and P. Fischer, Opt. Lett. 31, 453 (2006).

${ }^{6}$ D. J. Bornhop and J. Hankins, Anal. Chem. 68, 1677 (1996).

${ }^{7}$ A. Ghatak and K. Thyagarajan, Introduction to Fiber Optics (Cambridge University Press, Combridge, 1998), pp. 18, 384, 405.

${ }^{8} \mathrm{~J}$. McMurry, Organic Chemistry, 5th ed. (Brooks/Cole, Pacific Grove, CA, 2000), pp. 312-313.

${ }^{9}$ T. Dallas and P. K. Dasgupta, TrAC, Trends Anal. Chem. 23, 385 (2004).

${ }^{10}$ F. Pedrotti and L. Pedrotti, Introduction to Optics (Prentice-Hall, Englewood Cliffs, NJ, 1987), p. 353. 